\title{
Nanographite Films for Solid State Electronic Applications
}

\author{
Sergey G. Lebedev \\ Institute for Nuclear Research of Russian Academy of Sciences, 60th October Anniversary Prospect, 7a, Moscow 117312, Russia \\ Correspondence should be addressed to Sergey G. Lebedev; lebedev@inr.ru
}

Received 10 April 2013; Revised 17 June 2013; Accepted 14 July 2013

Academic Editor: Cristina Sbarra

Copyright (c) 2013 Sergey G. Lebedev. This is an open access article distributed under the Creative Commons Attribution License, which permits unrestricted use, distribution, and reproduction in any medium, provided the original work is properly cited.

\begin{abstract}
The structure and properties of nanographite films useful for applications in solid state devices are described. The possibility to use low conducting state of nanographite film for detecting radiation in the segmented solid state detectors is considered. Other interesting phenomena include the field effect conductivity switching which can be used in contactless current limiters and circuit breakers, the $r f$-to- $d c$ conversion which can be utilized in microwave and photo detectors, and light emitting subsequent to the conductivity switching with possible application as light sources. The possible underlying gears of the mentioned effects are discussed.
\end{abstract}

\section{Introduction}

Physical and electromagnetic properties of the carbon and its derivatives are the subjects of constant interest and extensive studies for many years. Attention has been paid to the conductivity switching behavior in the carbon graphite-like materials. Antonowicz et al. more than 30 years ago investigated the conductive properties of the glassy carbon [1] and its evaporated deposits [2] and has found out the effect of conductivity jump up to three orders of magnitude under critical current of the order of tens of milliamps. The change of conductivity was reversible, and the relaxation time was about some days. Later on, Antonowicz has revealed the change of samples current-voltage characteristics under irradiation of an $A l-C-A l$-sandwich with the microwaves [3]. Antonowicz has explained the effect observed by the superconductivity at room temperature [4].

Jang and Zhao also studied the switching behavior in carbonized materials [5]. They study the partially carbonized polyacrylonitrile fibers, which were observed to undergo a resistivity change between 2 and 4 orders of magnitude at a transition temperature typically in the range of $98^{\circ} \mathrm{C}$ to $200^{\circ} \mathrm{C}$. The current-voltage curves exhibited an initial supercurrentlike increase, followed by a rapid drop to a high resistance state, and then a rise in current again at a later stage. These phenomena cannot be interpreted by existing theories of switching in the inorganic amorphous semiconductors. They are explainable if the microstructure of the pyrolyzed fibers is viewed as comprising from the nanometer-scale superconducting phases interspersed with the semiconducting phases, much like a large number of the Josephson's junctions connected in series.

Goldberg et al. have obtained the US patent on the carbon conductivity switching device [6]. This device was made on the base of partially pyrolyzing polymer material by means of heating of the material to between $500^{\circ} \mathrm{C}$ and $800^{\circ} \mathrm{C}$. Electrodes are connected with the material at two different locations to produce an electrically active element therebetween. Devices made according to the teachings of the disclosure exhibit the negative resistance in a part of their voltagecurrent characteristic and run as the bidirectional electronic switches.

Over the past few years, there has been an increasing interest in applications of carbon-based films, such as diamond [7, 8], diamond-like carbon [9] and amorphous carbon $[10,11]$, and nanographite (NG) carbon [12] as the cathode materials in field emission display.

The author of this paper spent more than twenty years studying thin carbon films having in mind their application first as the strippers for the charge particle beams. Later on, the attention has been paid to the electromagnetic properties of thin carbon films. Some anomalies in electromagnetics of the films formed by sputtering of spectroscopic pure graphite in electrical arc discharge and also by chemical vapor deposition (CVD) methods have been found [13-16]. These were 


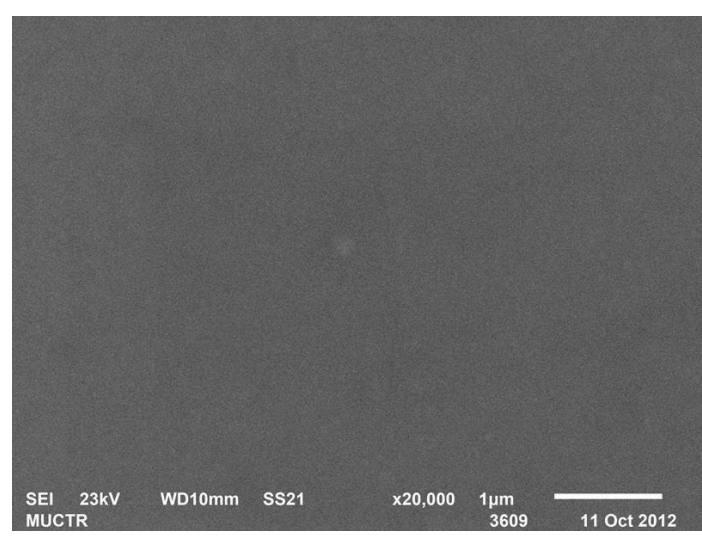

FIGURE 1: SEM image of typical nanographite film.

the field effect electrical resistivity jump, which can be used for current limiting in electric circuits and smart chips, the $r f$-to- $d c$ conversion, and the optical radiation emitted after conductivity switching off, which can be utilized in various optoelectronic devices.

Correlation of electrical and optical properties of NG films seems to be very promising in microelectronics. One of the examples of such correlations is the generation of lightinduced electric current in unbiased NG films [17]. The nature of electrooptical correlation so far is not so clean and will be discussed below.

The segmented solid state detectors are rapidly developing in many research fields including high energy physics at accelerators, astrophysics, and medicine $[18,19]$. Silicon very well fulfills the role of strip and pixel detectors. However, future detectors must provide fast signal collection, as the signal should be clearly related to one certain bunch crossing. Also, a prospective detector should cause a minimum of multiple scattering for the traversing particles. Thus, the detector must be kept as thin as possible. CVD diamond detector can meet some of these requirements [20]. In this paper, we report about switching behavior and electro-optical correlations observed in NG films and present some arguments about their advantages over diamond for using as strip and pixel detectors.

\section{Materials and Methods}

The nanographite films using in these experiments were obtained by means of CVD method on the quartz substrate. For thermal activation, gas mixture has been pumped in the one end sealed quartz tube which was placed in tubular oven. CVD process takes place in thermal activated hydrogenacetylene gas mixture at the temperature of $900^{\circ} \mathrm{C}$. As a result, the 1-micrometer thick NG film has been produced. Figure 1 represents typical scanning electron microscopy (SEM) image of NG film. As can be seen at highest magnification of 20000 , the surface of deposited film looks structureless which means the film is amorphous. This result is consistent with the representation [21] about NG films as a composite of small graphite-like $s p^{2}$-bonding granules embedded in the matrix

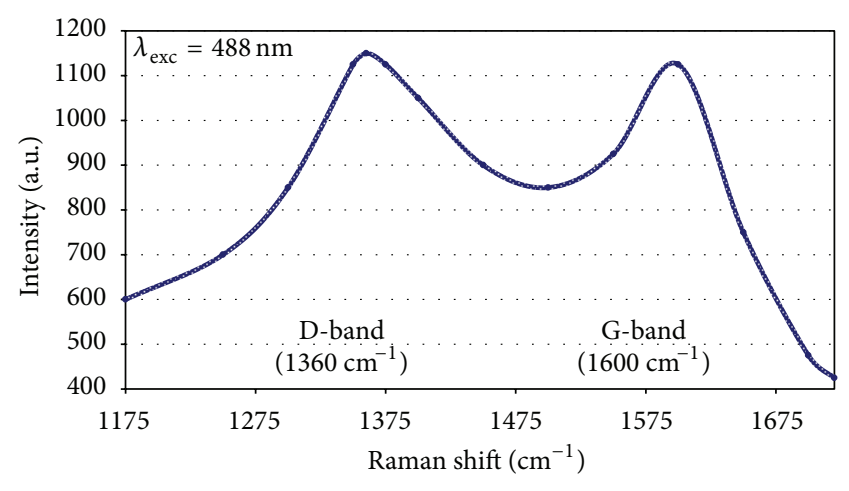

FIGURE 2: Raman spectrum of typical nanographite film.

of amorphous carbon. This conclusion is supported by in situ Raman spectroscopy.

Typical Raman spectrum of NG film is shown in Figure 2. In this spectrum, the Raman D-band at $1360 \mathrm{~cm}^{-1}$ and Gband in the vicinity of $1600 \mathrm{~cm}^{-1}$ is specific for different forms of disordered graphite. The height equality of $\mathrm{D}$ and $\mathrm{G}$ peaks corresponds to graphite crystalline size of the order 20-30 A [22].

As the measuring device, the four-channel digital oscilloscope LeCroy WaveRunner 6100 was used. The maximal frequency of digitization of $10 \mathrm{GHz}$ that allows to measure the processes in due course increases upto 225 picoseconds, a memory size on one channel-2 MB. As a source of a voltage the standard block of stabilized voltage supply with a limiting voltage of $100 \mathrm{~V}$ and a current of $250 \mathrm{~mA}$ was used.

For a programmable $I-V$ measurements, a Keithley 220 current source was used to supply a current, which was also monitored by a four-wire precision resistor of $0.1 \Omega$. The voltage drop on this resistor and on the sample was measured with a Keithley 2182 nanovoltmeter. An Agilent 34970 A Switch Unit, with a 34901A 20 Channel Multiplexer card and a 34970A48 Matrix Switch card, was used to allow for switching of both voltages and the current polarity, respectively, interconnecting the test leads with the nanovoltmeter and the current source in a break-before-make mode. All these instruments had IEEE-488.2 interfaces and used an IBMcompatible personal computer as the controller.

For the conductive measurements, we used the samples of $1-2 \mathrm{~cm}^{2}$ square with copper current leads arranged in planar geometry and connected to film surface by means of a silver paste. $I-V$ and conductivity switching characteristics of these samples were studied using both programmable and manual controlling current source.

\section{Results and Discussion}

The current-voltage and electrical switching characteristics of NG film samples are shown in Figure 3. In the "ON" state with the increasing of voltage, the current through the sample initially increases linearly (Figure 3). Near the critical current, the samples show nonlinearity. At the critical current, the samples exhibited a jump into metastable "OFF" state with the resistivity of four to five orders of magnitude higher of that 


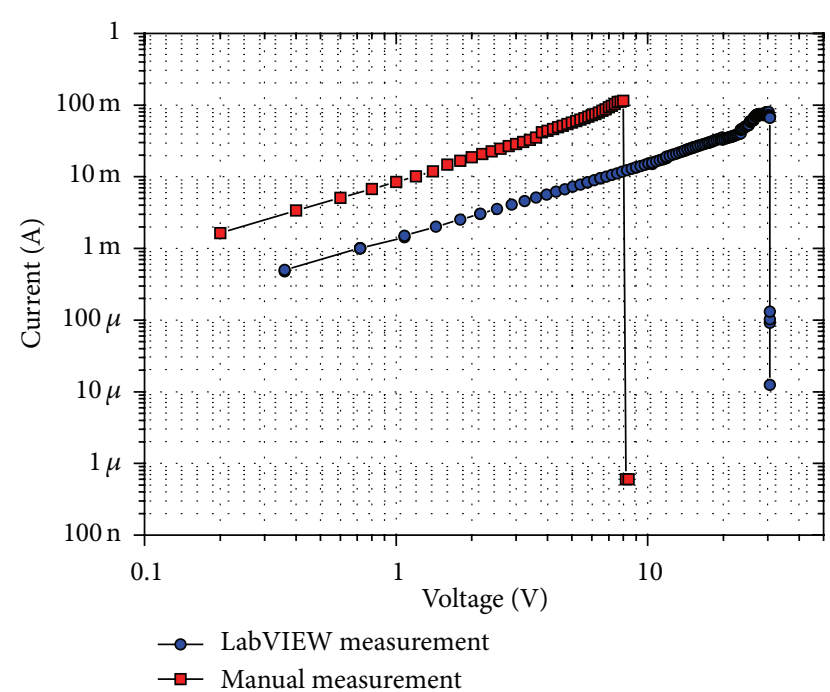

FIGURE 3: $I-V$ characteristics of 1 micrometer layer of NG film on quartz substrate.

into "ON" state. When the applied voltage decreased to zero, the sample reverts into the "ON" state and the switcher can be used repeatedly many times.

The switching rate measurement has been made by oscilloscope on load resistance. The switching process is shown in Figure 4. As can be seen, the duration of switching process is about 200 microseconds. This value varies up to $50 \%$ from sample to sample.

It is interesting to note that after current termination in "OFF" state, the light emission takes place. Some details are published elsewhere [23]. In support of this observation, the light emission has been observed also in relation with conductivity switching effects in semiconducting carbon fibres [24].

Such switching behavior is drastically different from other conductivity switchers described elsewhere [24-26]. The essential of all of these devices is switching from the low conducting (LC) to high conducting (HC) state. For explanation of such kind of switching, numerous hypotheses have been proposed: thermal and double injection mechanism [27], exciton's mechanism [24], and bistability caused by the overheating of electrons $[28,29]$. In our opinions none of these hypotheses can explain our results because in our case the switching occurs from HC to LC state that is a reverse to the processes mentioned previously.

Thermal mechanism fails to explain our results due to fast switching with "electronic" times of 100-200 microseconds (see Figure 4). Also, we observed optical radiation emitted few milliseconds after switching off. It is believed that this process is related with the thermocooling of sample after switching.

Double injection mechanism suggests the existence of trap centers in the structure. Saturation of these centers with injected curriers gives rise to jump into LC state. However, it is not possible to reverse this process to produce $\mathrm{LC}$ to $\mathrm{HC}$ transition.

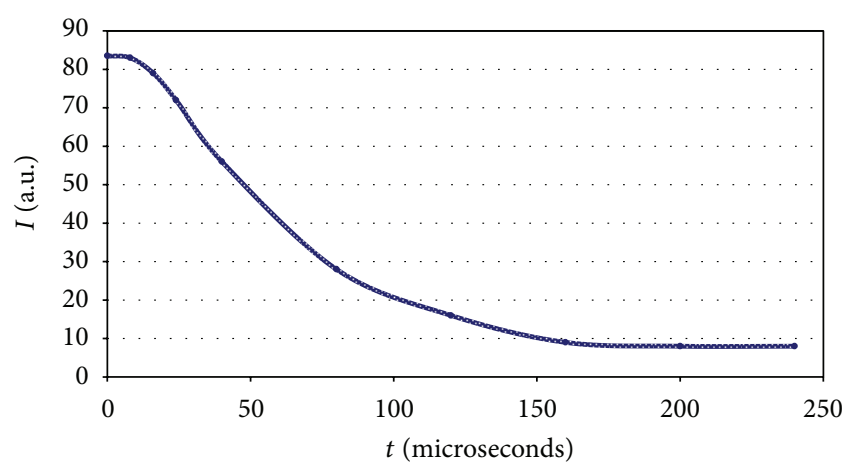

FIGURE 4: Switching process in NG film sample.

As for the hypothesis of hot electrons, the steep change of resistivity on few orders of magnitude should be located in near vicinity of working temperature. In the case described in $[28,29]$, this is the superconductor-insulator transition near the critical temperature. In [5], the evidence of steep metal-semiconductor transition between $\mathrm{HC}$ and $\mathrm{LC}$ around a temperature of $425^{\circ} \mathrm{K}$ is presented. In our case, there are no definite proofs for the existence of such a kind of transition around room temperature.

The superconducting hypothesis [4] seems to be promising in explaining the results obtained in $[1-3]$ as well as the switching behavior of NG films and our results described elsewhere [13-16]. Of course this hypothesis now is far from its proof. First of all, the superconducting behavior should be related to steep transition into zero resistive (or high conductive) state. Though the metal-insulator transitions in graphite [30-32] and disordered carbon [33,34] are well known, as it has been mentioned before, so far in NG films, we have not found steep transition into insulating state above room temperature. But the main disadvantages of the superconducting hypothesis are the absence of zero resistance state and the Meissner effect in all experiments mentioned previously. However, both contradictions can be explained by movement of magnetic flux quanta in the type II superconductor with sufficiently small lower critical magnetic field.

Another interesting feature of NG films is the $A C$-to$D C$ conversion and its temperature dependence presented in Figure 5. We have observed the effect of microwave detection as the occurrence of $D C$ voltage under $A C$ microwave irradiation. The temperature dependence of $V_{\mathrm{DC}}$ was measured by using the electrical circuit of [28] at $V_{\mathrm{AC}}=10 \mathrm{~V}$ and $R F$ frequency $f=1 \mathrm{MHz}$. The value of a $D C$ voltage on the detector exponentially decreased with the increase in temperature of the sample and tends to be zero at $\sim 370^{\circ} \mathrm{C}$. For more details please see [35].

Such kind of rectification is similar to photovoltaic effect described in $[36,37]$, where it is found that irradiation of the nanographite film by nanosecond laser pulses produces the electric current in unbiased NG films. The photovoltaic effect was explained as follows. $s p^{2}$ hybridization of electron orbitals leads to two-dimensional ballistic transport of electrons in graphene sheets of NG films. At the wavelength of 2.5 micrometers $(\sim 100 \mathrm{THz})$, the change of signal polarity was 


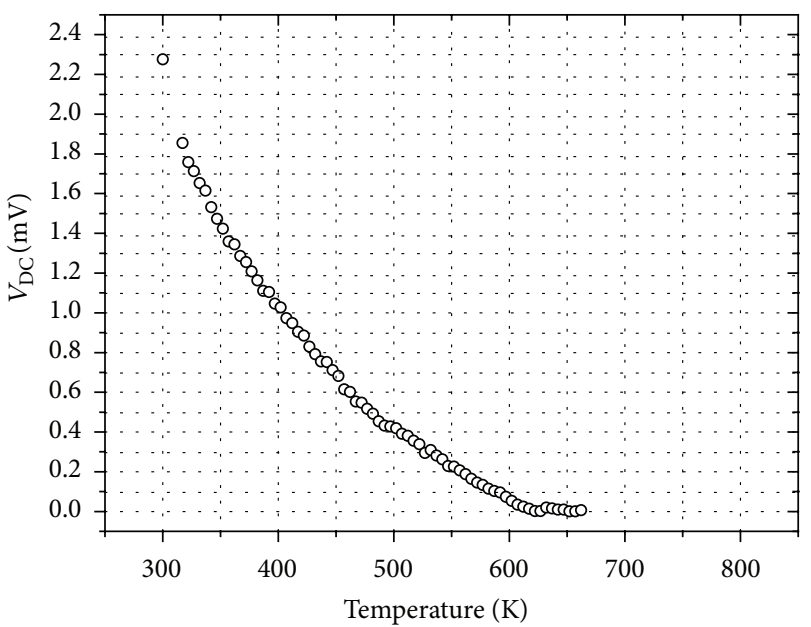

FIGURE 5: $A C$-to- $D C$ conversion in NG film.

observed [37]. This was explained by combination of two factors: photon drag (PDE) [38] and surface photogalvanic effects (SPGE) [39]. The photovoltaic effect is of interest for the development of high-speed photodetectors.

In contrast, the effect of $A C$-to- $D C$ conversion was observed in radiofrequency band. In this case, the change of $D C$ polarity takes place many times in the explored band width (see Figure 6) which cannot be explained by combination of PDE and SPGE. Moreover, polarity changes not only due to frequency change but also due to $A C$ voltage amplitude change.

The $A C$-to- $D C$ conversion of Figures 5 and 6 is principally different from more commonly known rectification effects due to polarity changes as functions of frequency (Figure 6) and $V_{\mathrm{AC}}$. This can be explained by the Josephson-like behavior based on the reversed Josephson effect $[13,16]$ according to the analogy with conventional [40] and multiphase granular superconductors [41]. In this case, for the dependence of $V_{\mathrm{DC}}$ on frequency $f$ and $V_{\mathrm{AC}}$, the following relation is fulfilled [42]:

$$
V_{\mathrm{DC}} \sim\left|J_{n}\left(\frac{2 e V_{\mathrm{AC}}}{\hbar f}\right)\right|,
$$

where $J_{n}$ is the $n$ th-order of Bessel function having many zeros. As can be seen from Figure 6 the relation (1) is likely to fulfill qualitatively in our case. There is no quantitative agreement due to a series combination of a large number of individual junctions whose individual-induced voltages vary in some random oscillatory manner as function of $f$ and $V_{\mathrm{AC}}$. Of course the mentioned Josephson explanation requires further confirmations.

The choice of material for segmented detector is very important. The advantages of solid state materials are efficiency, high density, room temperature operation, highly developed technology, robustness, and well suiting for microelectronics readout. Silicon, which is a standard material for vertex and tracking detectors in high energy physics, can operate at room temperature. Diamond (CVD or single crystal) has a large band gap and requires no depletion zone,

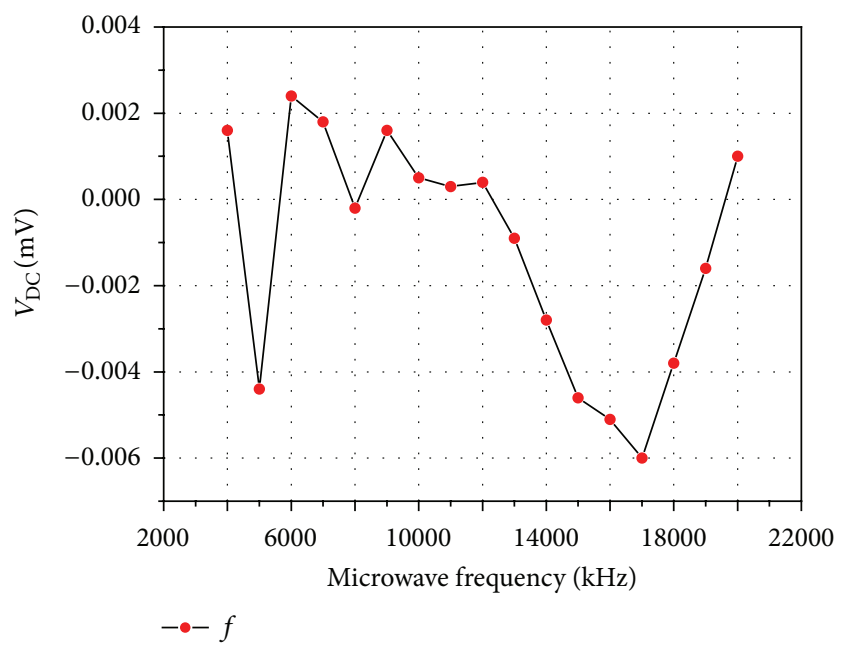

FIGURE 6: Frequency dependence of DC voltage induced by applied radiofrequency $\mathrm{AC}$ voltage.

very radiation hard. Drawback of diamond-like materials is a low signal and high cost. It is interesting to note that the value of band gap of NG materials varies with changing the ratio of $\mathrm{C}$ atoms in $\mathrm{sp}^{2}$ and $\mathrm{sp}^{3}$ coordinations. So, there is the possibility to choose detector material with optimal, in terms of specific objective, band gap. The advantage of NG materials over diamond is their better radiation stability. This is due to graphitization process in diamond materials under irradiation which gives rise to formation of crystalline graphite structure $[43,44]$.

Another possibility to use NG films in the segmented solid state detectors is using the conductivity switching behavior of Figure 3. The metastable high resistance state of NG film is characterized by low charge currier concentration which gives rise to small leakage current. As can be seen from Figure 3 in metastable OFF state, the NG film has a leakage current of about $\sim 1 \mu \mathrm{A}$ and the resistance of a few tens Mom. This current is dominated by thermally generated electronhole pairs. As it is known, the semiconductor's leakage current $I_{L}$ can be described as follows:

$$
I_{L} \sim T^{2 / 3} \exp \left(\frac{-E_{g}}{2 k T}\right),
$$

where $E_{g}$ is the band gap and $T$ is the temperature of detector material. According the relation (2), the band gap of NG film can be evaluated as about $1 \mathrm{eV}$ which is consistent with experimental data on band gap for NG family $0.5 \mathrm{eV}<E_{g}<1.5 \mathrm{eV}$ [45]. One of the most important parameter of a detector is the signal to noise ratio (SNR). The advantage of the discussed method is the absence of depleted zone and possibility of thin detecting layers of a few microns. This gives rise to SNR increasing up to 2-3 orders of magnitude for NG film as compared with bulk semiconductor.

\section{Conclusion}

To conclude the properties of NG films useful in solid state applications has been considered. Among them the field effect 
switching of conductivity which can be utilized in contactless current limiters of electrical circuits and smart chips, the $r f$ to- $d c$ conversion with possible application as the microwave and photo detectors, and light emitting subsequent to the switching of conductivity turning to account for light source applications. Some discussion of possible mechanisms of these effects has been done. The possibility to use the metastable OFF state of NG film for detecting radiation in the segmented solid state detectors has been discussed. It was shown that using NG film as a detector can increase the SNR up to 2-3 orders of magnitude as compared with bulk semiconductors.

\section{Acknowledgments}

This work was done with the support of the Russian Foundation for Basic Research (RFBR) under Grant no. 05-0817909-a. The author wishes to thank A. Saveliev for help in producing SEM and Raman measurements.

\section{References}

[1] K. Antonowicz, L. Cacha, and J. Turlo, "Switching phenomena in glassy carbon," Carbon, vol. 11, no. 1, pp. 1-5, 1973.

[2] K. Antonowicz, A. Jesmanowicz, and J. Wieczorek, "Switching phenomena in amorphous carbon," Carbon, vol. 10, no. 1, pp. 81-84, 1972.

[3] K. Antonowicz, "Effect of microwaves on DC current in an Alcarbon-Al sandwich," Physica Status Solidi A, vol. 28, no. 2, pp. 497-502, 1975.

[4] K. Antonowicz, "Possible superconductivity at room temperature," Nature, vol. 247, no. 5440, pp. 358-360, 1974.

[5] B. Z. Jang and L. Zhao, "Anomalous electrical behavior of partially carbonized polyacrylonitrile fibers," Journal of Materials Research, vol. 10, no. 10, pp. 2449-2453, 1995.

[6] H. A. Goldberg, I. L. Kalnin, C. C. Williams, and I. L. Spain, US Patent 4,642,664, 1987.

[7] M. W. Geis, J. C. Twichell, N. N. Efremow, K. Krohn, and T. M. Lyszczarz, "Comparison of electric field emission from nitrogen-doped, type Ib diamond, and boron-doped diamond," Applied Physics Letters, vol. 68, no. 16, pp. 2294-2296, 1996.

[8] S. Salvatori, E. Brugnoli, M. C. Rossi, and F. Pinzari, "Fieldand photo-emission properties of CVD-diamond with different microcrystalline structure," Diamond and Related Materials, vol. 10, no. 3-7, pp. 852-857, 2001.

[9] R. K. Roy, S. Gupta, B. Deb, and A. K. Pal, "Electron field emission properties of electro-deposited diamond-like carbon coatings," Vacuum, vol. 70, no. 4, pp. 543-549, 2003.

[10] M. T. Kuo, P. W. May, and M. N. R. Ashfold, "Field emission site density studies of amorphous carbon films," Diamond and Related Materials, vol. 11, no. 7, pp. 1422-1428, 2002.

[11] W. I. Miline, J. T. H. Tsai, and K. B. K. Teo, "Novel field emission structure based on tetrahedrally bonded amorphous carbon," Diamond Related Materials, vol. 12, no. 2, pp. 195-200, 2003.

[12] A. N. Obraztsov, A. P. Volkov, K. S. Nagovitsyn et al., "CVD growth and field emission properties of nanostructured carbon films," Journal of Physics D, vol. 35, no. 4, pp. 357-362, 2002.

[13] S. G. Lebedev and S. V. Topalov, "Evidence of weak superconductivity in carbon films," Bulletin of Lebedev's Physical Institute N, no. 11-12, pp. 14-21, 1994.
[14] S. G. Lebedev, "Particle irradiation for verification of superconducting-like behavior in carbon arc films," Nuclear Instruments and Methods in Physics Research A, vol. 521, no. 1, pp. 2226, 2004.

[15] S. G. Lebedev, V. E. Yants, and A. S. Lebedev, "Correlations between switching of conductivity and optical radiation observed in thin graphite-like films," Nuclear Instruments and Methods in Physics Research A, vol. 590, no. 1-3, pp. 227-233, 2008.

[16] S. G. Lebedev, "Evidence of Josephson-like behaviour of thin granular carbon films," International Review of Physics, vol. 2, p. 312, 2008.

[17] G. M. Mikheev, R. G. Zonov, A. N. Obraztsov, and Y. P. Svirko, "Giant optical rectification effect in nanocarbon films," Applied Physics Letters, vol. 84, no. 24, pp. 4854-4856, 2004.

[18] H. Spieler, Semiconductor Detector Systems, Oxford University Press, Oxford, UK, 2005.

[19] L. Rossi, P. Fischer, T. Rohe, and N. Wermes, Pixel Detectorsfrom Fundamentals to Applications, Springer, New York, NY, USA, 2006.

[20] M. Friedl, W. Adam, C. Bauer et al., "CVD diamond detectors for ionizing radiation," Nuclear Instruments and Methods in Physics Research, vol. 435, no. 1-2, pp. 194-201, 1999.

[21] C. Z. Wang, K. M. Ho, and C. T. Chan, "Tight-binding molecular-dynamics study of amorphous carbon," Physical Review Letters, vol. 70, no. 5, pp. 611-614, 1993.

[22] P. K. Chu and L. Li, "Characterization of amorphous and nanocrystalline carbon films," Materials Chemistry and Physics, vol. 96, no. 2-3, pp. 253-277, 2006.

[23] S. G. Lebedev, V. E. Yants, and A. S. Lebedev, "Correlations between switching of conductivity and optical radiation observed in thin graphite-like films," Nuclear Instruments and Methods in Physics Research A, vol. 590, no. 1-3, pp. 227-233, 2008.

[24] L. R. Zhao and B. Z. Jang, "Switching behaviours in semiconducting carbon fibres," Journal of Materials Science Letters, vol. 15, no. 2, pp. 99-101, 1996.

[25] S. R. Ovshinsky, "Reversible electrical switching phenomena in disordered structures," Physical Review Letters, vol. 21, no. 20, pp. 1450-1453, 1968.

[26] S. S. K. Titus, R. Chatterjee, S. Asokan, and A. Kumar, "Electrical switching and short-range order in As-Te glasses," Physical Review B, vol. 48, no. 19, pp. 14650-14652, 1993.

[27] N. F. Mott, "Conduction in non-crystalline systems VII. Nonohmic behaviour and switching," Philosophical Magazine, vol. 24, no. 190, pp. 911-934, 1971.

[28] B. L. Altshuler, V. E. Kravtsov, I. V. Lerner, and I. L. Aleiner, "Jumps in current-voltage characteristics in disordered films," Physical Review Letters, vol. 102, no. 17, Article ID 176803, pp. 803-806, 2009.

[29] M. Ovadia, B. Sacepe, and D. Shahar, "Electron-phonon decoupling in disordered insulators," Physical Review Letters, vol. 102, no. 17, pp. 176802-176803, 2009.

[30] J. C. González, M. Muñoz, N. García et al., "Sample-size effects in the magnetoresistance of graphite," Physical Review Letters, vol. 99, no. 21, Article ID 216601, 2007.

[31] G. Timp, P. D. Dresselhaus, T. C. Chieu, G. Dresselhaus, and Y. Iye, "Anomalous magnetoresistance of graphite at high magnetic fields," Physical Review B, vol. 28, no. 12, pp. 7393-7396, 1983. 
[32] Z. M. Wang, Q. Y. Xu, G. Ni, and Y. W. Du, "Huge magnetoresistance and Shubnikov-de Hass effect in graphite," Physics Letters, vol. 314, no. 4, pp. 328-331, 2003.

[33] K. Kuriyama and M. S. Dresselhaus, "Metal-insulator transition in highly disordered carbon fibers," Journal of Materials Research, vol. 7, no. 4, pp. 940-945, 1992.

[34] A. W. P. Fung, Z. H. Wang, M. S. Dresselhaus, G. Dresselhaus, R. W. Pekala, and M. Endo, "Coulomb-gap magnetotransport in granular and porous carbon structures," Physical Review B, vol. 49, no. 24, pp. 17325-17335, 1994.

[35] S. G. Lebedev, Ed., Unconventional Electromagnetics in Carbonaceous Materials, Nova Science Publishers, Huntington, NY, USA, 2010.

[36] G. M. Mikheev, V. M. Styapshin, P. A. Obraztsov, E. A. Khestanova, and S. V. Garnov, "Effect of laser light polarisation on the dc photovoltage response of nanographite films," Quantum Electronics, vol. 40, no. 5, pp. 425-430, 2010.

[37] P. A. Obraztsov, G. M. Mikheev, S. V. Garnov, A. N. Obraztsov, and Y. P. Svirko, "Polarization-sensitive photoresponse of nanographite," Applied Physics Letters, vol. 98, no. 9, Article ID 091903, 2011.

[38] V. L. Al'perovich, V. I. Belinicher, V. N. Novikov, and A. S. Terekhov, "Surface photovoltaic effect in solids. Theory and experiment for interband transitions in gallium arsenide," Journal of Experimental and Theoretical Physics, vol. 53, no. 6, p. 1201, 1981.

[39] V. L. Gurevich and R. Laiho, "Photomagnetism of metals. First observation of dependence on polarization of light," Physics of the Solid State, vol. 42, no. 10, pp. 1807-1812, 2000.

[40] H. Sadate-Akhavi, J. T. Chen, A. M. Kadin, J. E. Keem, and S. R. Ovshinsky, "Observation of rf-induced dc voltages in sputtered binary superconducting films," Solid State Communications, vol. 50, no. 11, pp. 975-978, 1984.

[41] J. T. Chen, L. E. Wenger, C. J. McEwan, and E. M. Logothetis, "Observation of the Reverse ac Josephson Effect in Y-Ba-Cu-O at 240 K," Physical Review Letters, vol. 58, no. 19, pp. 1972-1975, 1987.

[42] J. T. Chen, R. J. Todd, and Y. W. Kim, "Investigation of microwave-induced dc voltages across unbiased Josephson tunnel junctions," Physical Review B, vol. 5, no. 5, pp. 1843-1849, 1972.

[43] E. A. Koptelov, S. G. Lebedev, and V. N. Panchenko, "A model of carbon stripper target failure under ion beam bombardment," Nuclear Inst. and Methods in Physics Research, A, vol. 256, no. 2, pp. 247-250, 1987.

[44] E. A. Koptelov, S. G. Lebedev, and V. N. Panchenko, "Radiation stability of carbon foil microstructure," Nuclear Instruments and Methods in Physics Research B, vol. 42, no. 2, pp. 239-244, 1989.

[45] J. Robertson, "Diamond-like amorphous carbon," Materials Science and Engineering R, vol. 37, pp. 129-281, 2002. 

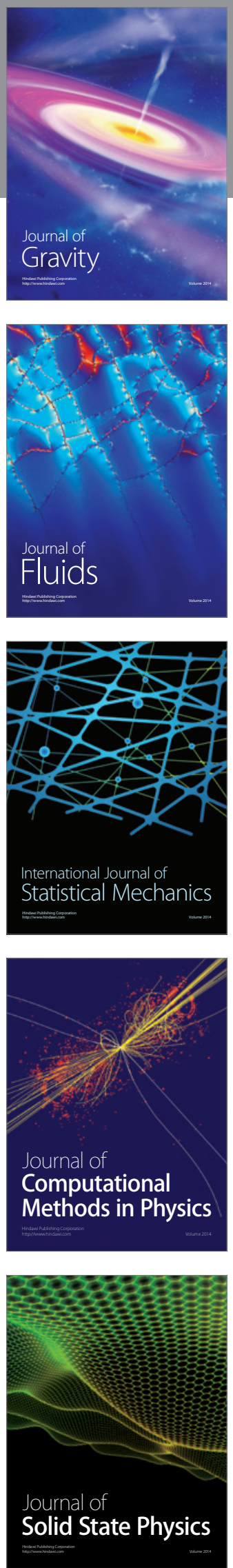

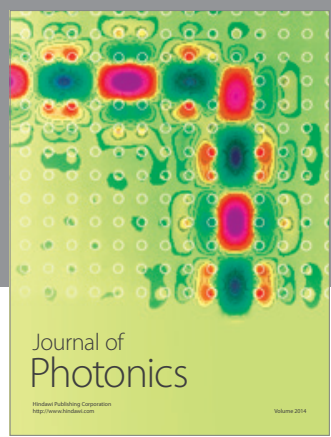

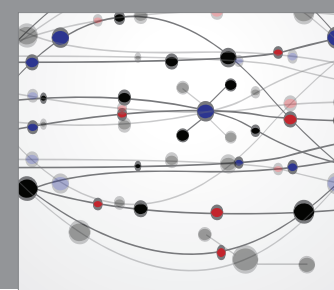

The Scientific World Journal

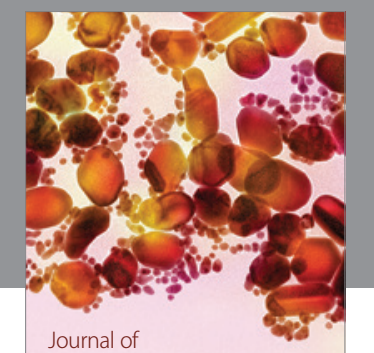

Soft Matter
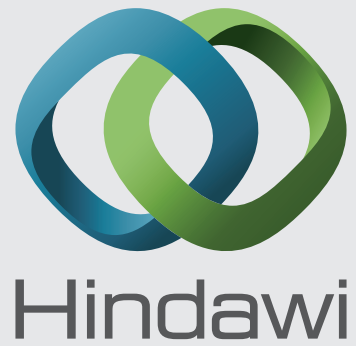

Submit your manuscripts at

http://www.hindawi.com
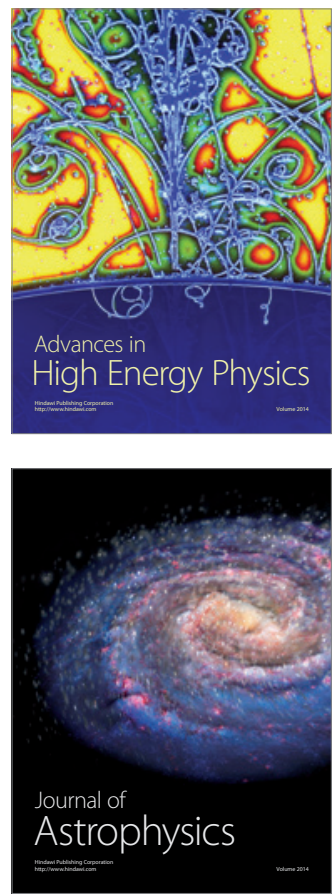
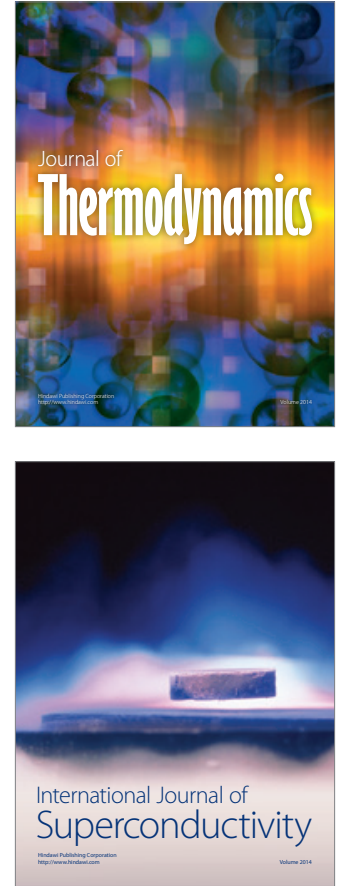
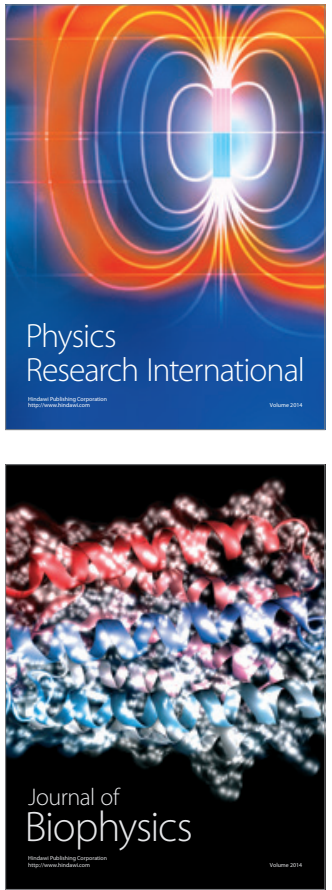
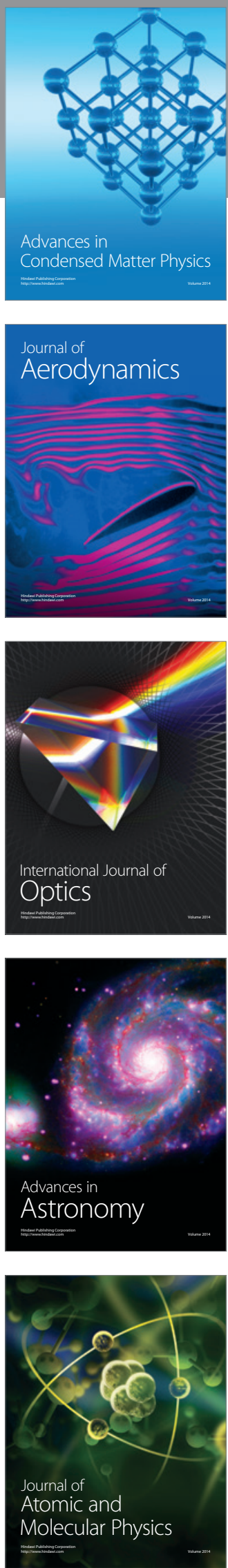